\title{
Crise du français, impact de la francophonie?
}

\author{
Oana Panaïté \\ Indiana University-Bloomington
}

Peut-on parler d'une crise actuelle du français, de la langue comme de la discipline dont elle est le vecteur, communément appelée dans les milieux universitaires étasuniens et britanniques les «études françaises »? Assiste-t-on cependant à un impact grandissant de la francophonie, c'est-àdire des formes linguistiques, littéraires et culturelles hybrides ? Ce double mouvement est-il bénéfique ou cache-t-il un dangereux travail de sape aussi bien intellectuel qu'institutionnel ?

Ces quelques questions formaient le bref argumentaire de l'appel à contributions pour la session spéciale du Conseil International d'Études Francophones au congrès annuel de la Modern Language Association, organisé en 2014 à Chicago.

Il est impossible de séparer le débat sur la précarisation du français au sein de l'institution universitaire américaine (Simon) de la dépréciation économique, institutionnelle et axiologique touchant les humanités non seulement aux États-Unis, mais aussi en Europe, y compris en France. Selon certains critiques du phénomène, les fonctions morales et sociales attribuées aux lettres modernes et classiques - passion du savoir et de la recherche, admiration désintéressée du beau, perfectionnement individuel grâce à l'étude des classiques - se voient décriées sous la pression des logiques marchandes, des décisions politiques conditionnées par les comportements clientélaires et consuméristes et de l'apathie intellectuelle chronique dans le rang des étudiants (Edmunson 10) ${ }^{1}$. Cependant, d'autres analyses signalent que les jérémiades issues de "l'idéologie victimaire » (Gans 40) ${ }^{2}$ font long feu devant une situation historique amorcée au dixhuitième siècle et correspondant à une dévalorisation progressive que la

\footnotetext{
${ }^{1}$ Dans le domaine français on peut renvoyer aux travaux d'Yves Citton qui articulent une réflexion sur la fonction politique de la littérature avec la théorie littéraire, notamment l'étude du récit et de la fiction.

2 Après avoir dressé un parallèle entre la pratique contemporaine du blogue et La Recherche $d u$ temps perdu, Gans affirme dans la conclusion de son article: "Yet a world where the literary myth survives only in muted forms is not a world without literature, merely a world where literature knows its place, a world of stories that we need not believe in as the universal story. » (Gans 41)
} 
littérature reflète et à laquelle elle-même réfléchit avec obstination depuis plusieurs siècles. L'absolutisation presque religieuse de la littérature à l'ère de son expansion de pair avec l'autonomisation de sa sphère esthétique et ontologique ont donc participé à une survalorisation responsable de sa dépréciation actuelle. Pour Peter Sloterdijk, «la littérature, la correspondance et l'idéologie humaniste n'influencent plus aujourd'hui que marginalement les mégasociétés modernes dans la production du lien politico-culturel. Ce n'est nullement la fin de la littérature. Mais elle s'est marginalisée en une subculture sui generis et le temps où elle était surestimée comme vecteur de l'esprit national n'est plus » (Sloterdijk viii). Si ces débats font ressortir les contradictions politiques et axiologiques de la société contemporaine, ils mettent aussi à mal la vision autonome ou autotélique de la littérature face aux demandes de la vie quotidienne.

Envisagée sous cet angle, la menace qui pèse sur la littérature marquerait une fin salutaire, et ce, pour deux raisons. Premièrement, parce qu'elle clôt une période de «mainmise sur la culture par les études littéraires » (Appadurai 95). Deuxièmement, parce que la fin tant débattue de la littérature - de ses institutions traditionnelles, de ses fonctions sociales et de son prestige culturel - s'accompagne de l'élargissement des champs disciplinaires et de l'éclosion des recherches nouvelles. Ceux qui s'emparent du discours de la fin, poussant la réflexion au-delà de la rhétorique de la lamentation, y trouvent un terrain fertile pour transmuer l'épuisement en un concept porteur de sens et catalyseur de nouvelles perspectives.

Les principes fondateurs et la pertinence historique du système de valeurs littéraires qui trouve son expression institutionnelle dans les Classiques ou le canon, catégorie générique réunissant auteurs et œuvres associés, au terme d'un processus historique de réévaluation et de redistribution symbolique, à un idéal de perfection, et participant en retour à la légitimation du système auquel ils fournissent des modèles exemplaires. Ainsi que le montrent les travaux sur ce que Paul Bénichou appelle «la dignification de la littérature profane » (Benichou 13), dans l'invention de la culture aux $\mathrm{XVII}^{\mathrm{e}}$ et $\mathrm{XVIII}^{\mathrm{e}}$ siècles, la littérature joue un rôle double "puisqu'elle donne une assise imaginaire et un mode de communication aux nouvelles pratiques de sociabilité en même temps qu'elle reçoit de cette 'sphère culturelle' une publicité et une valeur incontournables » (Méchoulan 27). La question est alors de savoir si les caractéristiques de l'œuvre ou de l'auteur classique ainsi qu'elles ont été formulées durant les périodes d'émergence et d'institutionnalisation de la littérature rendent comptent des transformations historiques, géographiques et sociales qui infléchissent la 
production et la réception littéraire moderne. Les réponses varient selon que l'on a recours à une définition esthétique, qui fait du style le trait dominant et lui subordonne valeur morale ou qualité intellectuelle ou à une définition idéologique qui fait du classique l'expression du caractère national. Des initiatives telles que les études transhistoriques et transfrontalières peuvent être perçues, d'une part, comme participant d'une production de récits consolateurs et, d'autre part, comme une relance critique. Si l'on choisit de suivre la seconde hypothèse, on s'aperçoit que le pessimisme aux résonances parfois apocalyptiques à l'égard de la littérature concerne en fait un certain nombre de conceptions théoriques et de paradigmes herméneutiques, autrement dit son péritexte interprétatif ou les études littéraires ${ }^{3}$.

À cet aggiornamento de la pensée sur la littérature qui combine l'hétérogénéité post-disciplinaire avec la souplesse conceptuelle, semble correspondre une entropie créatrice de la pensée de la littérature. Car, suivant l'étiolement des formalismes et le discrédit des poétiques collectives, la réflexion et la pratique littéraire reflètent le refus de l'embrigadement sans toutefois sacrifier le rêve d'une communauté qu'elles reconstituent de manière souple et discrète, à partir d'affinités électives et de préoccupations partagées.

S'inscrivant dans une mouvance qui vise à transcender ces divisions conceptuelles, French Global allègue de la nature transfrontalière de la tradition littéraire en français pour étayer une nouvelle approche critique. Après avoir souligné le caractère novateur des histoires de la littérature écrites en dehors de la France, mouvement critique inauguré par le travail que dirigea Denis Hollier en 1989, Christie McDonald et Susan Rubin Suleiman proposent une approche reposant sur la diversité des espaces, la multiplicité des formes et la mobilité des imaginaires. L'idée d'une littérature française homogène, miroir de l'esprit national, éclate sous la pression de ses contradictions internes et de ses impensés identitaires: "Our contention is that such questions - about the tension between the multiplicity and unity, between diversity and uniformity, between 'same' and 'other', as well as the related questions of migration and diasporic

\footnotetext{
${ }^{3}$ Dans la vaste bibliographie portant sur le sujet, l'on retiendra la synthèse offerte par la revue numérique Fabula. Littérature, Histoire, Théorie sous le titre «Le partage des disciplines », 8 (2011) (www.fabula.org/lht/8/), préfacé par l'article de Nathalie Kremer, «La littérature en mal de discipline ». Ce numéro rassemble les interventions de François Hartog, Jan Baetens, Michel Collot, Stanley Fish et Ronald Shusterman, entre autres, sur des sujets aussi divers que la défense culturelle des études littéraires, la géographie littéraire ou les écueils de l’interdisciplinarité.
} 
identities - are not limited to the emergence of 'Francophone literature'; rather, they have informed every period of French literature, starting with some of its most canonical texts » (McDonald et Suleiman xi).

Séparés par une tradition disciplinaire et institutionnelle qui envisage différemment la fonction, les valeurs et jusqu'à la définition de la littérature selon le contexte historique qui les a vu naitre, les textes euxmêmes résistent pourtant à une division artificielle que ni auteurs, ni lecteurs, ni enseignants et, par ailleurs, ni la plupart des critiques n'adoptent dans leurs pratiques d'écriture, de lecture, d'enseignement et de recherche. Toutefois, entérinée dans les histoires littéraires et reprise par les manuels et programmes scolaires, la division temporelle et spatiale persiste, en érigeant la chronologie au rang de critère distinctif central et la distance géographique en catégorie définitoire déterminante. D'une part, les œuvres canoniques sont placées dans un panthéon qui, tout en les rendant exemplaires, les isole du vaste ensemble de la production littéraire auquel elles n'ont pourtant jamais cessé d'appartenir; d'autre part, les écritures contemporaines sont reléguées au rang transitoire d'ouvrages à valeur artistique variable, soit trop élitistes et inaccessibles, expression d'une attitude poétique expérimentale, soit trop conventionnelles et commerciales, émanant d'une volonté de se conformer aux attentes du public. La conséquence de cette dissymétrie dans les pratiques d'enseignement est souvent paradoxale: une survalorisation sans fréquentation des classiques et une surreprésentation sans évaluation des contemporains. Alors que ce chassé-croisé conduit à l'approfondissement des clivages disciplinaires, notamment de celui qui oppose la littérature «française » aux littératures "francophones », il est nécessaire d'innover la réflexion théorique et l'étude scolaire des textes à partir d'une vision globale, dynamique et multipolaire de la littérature en français.

L'émergence des littératures en français écrites hors de l'Hexagone et la visibilité croissante de leurs auteurs, invitent étudiants, enseignants et chercheurs à élargir leur horizon culturel, à repenser les hiérarchies artistiques, à innover leur outillage conceptuel et à imaginer de nouvelles façons de lire et de se situer par rapport aux textes. Cette nouvelle attitude permettrait d'envisager une constellation de relations entre passé et présent, « ici » et « ailleurs », création et réception.

On constate, dans les travaux récents d'histoire littéraire parus en France, l'absence des écritures francophones reflétant le désintéressement d'une certaine doxa académique française à l'égard d'une réalité aux formes fluides et variables. Les raisons de cette absence sont parfois formulées de 
manière sommaire, d'autant plus définitive qu'elle semble relever de l'évidence même. Dans l'Histoire de la littérature française du XX siècle publiée sous la direction de Michèle Touret, l'on trouve l'argument suivant: «La notion de francophonie, tardivement apparue dans le contexte politique de la décolonisation, recouvre des situations diverses qu'on ne saurait assimiler. Nous n'aborderons pas ici ce domaine aux frontières instables » (Touret 253). Pour brouiller davantage les cartes, à l'émergence des études postcoloniales s'oppose le partage disciplinaire mis en place depuis les années 1980, entre littérature française, d'une part, et littératures francophones, de l'autre. «Le français est une langue partagée. Plusieurs littératures, très différentes entre elles, l'écrivent» (Viart et Vercier 7), affirme l'introduction de La littérature française au présent, signée par Dominique Viart et Bruno Vercier. Pour justifier l'exclusion des écrivains non hexagonaux (y compris de ceux issus des régions et départements d'outre-mer), les auteurs de l'ouvrage s'appuient invoquent l'extrême diversité des cadres géographiques, historiques, politiques, ethniques et culturelles des littératures écrites en français: «Quel critère décide qu'un écrivain est français plutôt que francophone? sa naissance ? sa résidence? son éditeur originel ? sa nationalité ? Toutes choses - ou presque - qui peuvent changer. Et qui changent de fait. La géographie et l'histoire politique nous ont légué à cet égard des partages étonnants : on est français à la Guadeloupe et à la Réunion, mais francophone à Haïti et à Maurice, îles plus proches entre elles que la France métropolitaine. » (Viart et Vercier 8) Par souci d'intégrité scientifique, la lecture commune des écritures en français est à éviter: mal renseignée, un tel travail n'aboutira qu'au traitement superficiel et purement symbolique des littératures francophones auxquelles on se contente de faire "portion congrue ». Judicieuses en apparence, ces objections contre la prise en compte de la production non hexagonale dans un ouvrage consacré au dernier quart du vingtième siècle révèlent en réalité une "gêne épistémologique " (Panaïté 147) à l'égard de l'impensé critique des catégories telles que la classe, la race et la domination politique. Cet impensé est pris en charge par les études postcoloniales. Suivant la définition que B. Ashcroft, G. Griffith et H. Tiffin assignent à l'objet postcolonial dans l'introduction à l'ouvrage The Postcolonial Study Reader, à savoir un "produit culturel marqué par l'influence du colonialisme sans tenir compte du contexte particulier ou du contexte social dans lequel il fut élaboré »(Ashcroft, Griffith et Tiffin 5), la littérature française comme les littératures francophones s'inscrivent dans cette catégorie. Cependant, nombreux sont ceux qui remettent en question non seulement le rapport entre le cadre conceptuel postcolonial et les problèmes de la francophonie, mais encore l'existence même de ce rapport. Lydie Moudileno demande: «Comment la critique postcoloniale peut-elle 
être véritablement en phase avec des auteurs contemporains qui essayent justement d'échapper aux catégories?» (Moudileno). En soulignant le parallèle entre deux types de paramètres : l'un, identitaire, fondé sur des catégories historiques, générationnelles, géographiques, l'autre, esthétique, axé sur une logique de l'autonomie du processus artistique, l'on se prémunit contre l'écueil d'une fausse interprétation du terme «postcolonial» sous l'angle de la continuité avec l'époque chronologiquement précédente. Est-il utile d'adopter une approche esthétique fidèle aux revendications d'un statut transculturel et fondamentalement littéraire formulées par les écrivains eux-mêmes («nous sommes des écrivains tout court») et, si oui, à partir de quelles conditions et de quels critères peut-on lire ensemble des écrivains contemporains qui présentent cependant de fortes différences stylistiques, thématiques et idéologiques?

Il s'avère ainsi que les questions sur la signification et l'importance des études postcoloniales en cachent d'autres qui dépassent les frontières nationales, culturelles ou universitaires. Elles touchent aux principes fondateurs de la critique et de l'histoire littéraires. Quel est l'objet de la critique littéraire: l'analyse des œuvres singulières ou l'étude des phénomènes généraux (historiques, sociaux, politiques, formels) dans lesquels celles-ci s'inscrivent? Une approche théorique ne peut-elle se construire qu'aux dépens de la spécificité irréductible des objets étudiés ? Dans le contexte particulier de la littérature de langue française, comment doit-on aborder, sur le plan des méthodes aussi bien que des institutions, le décalage entre une conception géopolitique du monde francophone et par conséquent de sa littérature, dont le trait le plus saillant est la politique identitaire, d'un côté, et l'émergence d'un espace commun (koinè) investi par les écrivains qui partagent, dépassent, transforment et recréent la langue française, de l'autre? Il est possible d'envisager que la solution suppose l'élaboration de nouveaux rapports entre approches et disciplines telles que les études postcoloniales, la littérature française et les littératures francophones. C'est le défi lancé en 2003 par Mireille Rosello qui invite à renverser le rapport d'appartenance traditionnel entre la littérature française et la littérature francophone en intégrant celle-là au cadre général établit par celle-ci. Ce geste aux conséquences considérables amène d'abord une transformation de la francophonie elle-même, en la libérant de ses vieilles attaches politiques, d'un côté, et de la mauvaise conscience néocoloniale, de l'autre: "The desire to move away from two definitions of Francophonie: one that would imagine a hegemonic status of French linked to a colonial power as if by some sort of perverse umbilical cord, and a second definition that would suspect non-European Francophone writers of 
alienation if they choose to write in French » (Rosello 131-132). Concevoir la littérature hexagonale comme une branche de la francophonie conduit ensuite à un " unhoming » de la discipline littéraire. L'auteure de Postcolonial Hospitality appelle de ses vœux ce dépaysement engendré par les rencontres transnationales et transdisciplinaires qui puisse renouveler les principes fondateurs et les attitudes à l'égard de l'appartenance territoriale et de la légitimité nationale: «I would hope that such transnational and transdisciplinary encounters between types of Francophone studies would lead to a sort of 'unhoming' of the field : it would make us perceive our discipline not as 'homeless' [...] nor exiled (home is not somewhere else), but a struggling with unhomeliness, where legitimacy is a ghost that we keep conjuring up » (Rosello 132). C'est le lien constitutif entre deux gestes critiques qui est en jeu ici : réexaminer, d'une part, les concepts et méthodes de la critique postcoloniale afin de retracer, d'autre part, les frontières de l'objet mis en lumière par cette même critique. Prendre conscience du fait que le savoir littéraire émanant de la nouvelle relation entre les œuvres classiques et contemporaines, hexagonales et mondiales n'est ni sans-pays, flottant dans une globalisation indifférenciée, ni exilé, voué au déchirement perpétuel, revient aussi à rendre intelligible l'éthos de cette relation.

Le concept de cosmopolitisme, tel qu'il est réactualisé dans la pensée contemporaine (dans les travaux d'Arjun Appadurai, d'Étienne Balibar ou de Pheng Cheah), éclaire cette question d'une manière innovatrice. Défini autrefois comme un sentiment d'appartenance (religieuse, politique ou culturelle) au monde entier ou à la communauté humaine, il renvoie dans le contexte contemporain à l'idée d'une universalité humaine et à des formes de solidarité et même de résistance aux effets funestes de la globalisation. Parmi ces formes de résistance, l'idée et les pratiques de la littérature-monde s'avèrent particulièrement significatives pour notre débat sur la crise du français et l'impact de la francophonie. J'ai analysé ailleurs les manifestations poétiques et les enjeux politiques que cette idée révèle, mais qu'elle dissimule aussi (Panaïté 2012). Au-delà des effets de mode et des polémiques stériles, les travaux de Pheng Cheah offrent un survol historique et philosophique de l'idée de littératuremonde tout en soulignant sa pertinence actuelle pour la pensée du cosmopolitisme postcolonial. En soulignant la potentialité de la littérature lorsqu'elle agit comme un contre-pouvoir spécifiquement humain face à la logique marchande de la globalisation. Au sens philosophique, cette littérature ouvre le domaine des possibles et l'actualisation d'autres mondes auxquels celui, exclusif, de la globalisation néolibérale en vient à se heurter : «a particular type of postcolonial literature [...] explores the various 
negotiations between commercial and financial flows and humane social development [...] with the hope of crafting new figurations and stories of world-belonging for a given postcolonial people »(Chea 36). Il invite également à reconsidérer sens même du terme «monde », à partir de Kant, Goethe et Marx. Chez Kant, l'idée moderne de cosmopolitisme s'articule autour d'une conception du monde en tant que communauté globale des êtres humains à l'intérieur de laquelle l'individu, conscient de l'importance des autres, renonce à l'affirmation de ses intérêts au nom de la responsabilité collective. Dans un tel contexte ontologique et moral, la littérature permet l'avènement de l'humanité grâce à sa dimension sociale: « [it] brings humanity into being because it leads to sociability » (Cheah 27). Pour Goethe, la littérature mondiale (Weltliteratur) représente « an active space of transaction and interrelation » (Cheah 28); la notion de transaction étant délestée de ses connotations économiques, elle renvoie à un certain type de rapports entre nations qui suppose la coexistence, l'interaction et l'entente réciproque. Les agents privilégiés en sont les traducteurs, puisque leur travail, foncièrement créateur, facilite la circulation et le partage des valeurs communes à travers les frontières. Attelée à cette tâche universelle, la traduction assure en même temps le respect des particularités nationales qui composent le monde. L'intervention de Marx consiste, selon Cheah, à relever la dimension matérielle du monde, complémentaire de sa dimension géographique, au prisme de laquelle la littérature apparaît comme un simple épiphénomène. Face aux antagonismes mis en place par le système capitaliste, la littérature ne peut remplir qu'une fonction idéologique, de superstructure, sans pouvoir efficace, étant un produit parmi d'autres du système marchand. Toutefois Cheah infléchit la signification du «monde» en termes heideggériens, rejetant la simple synonymie avec le «globe» ou la " planète » en raison du statisme et de la finitude de ceux-ci qui s'opposent la processualité infinie de celui-là: « [t] he globe [is] the totality produced by processes of globalization »(Cheah 30), alors que le monde est « an ongoing process of becoming, something continually made and remade rather than a spatial-geographical entity » (Cheah 31). C'est précisément le statut ontologique infini, toujours et encore à faire, du monde conçu en tant que communauté inachevée que la littérature-monde est appelée à prendre en charge lorsqu'elle s'avère être une alternative à la réalité géographique, économique et politique que l'idéologie mondialiste tente d'imposer à l'exclusion de tout autre: " an inexhaustible resource for contesting the world given to us through commercial intercourse, monetary transactions, and the space-time compression of the global culture industry »(Cheah 36). 
Si les liens entre le cosmopolitisme et la littérature-monde, d'une part, et la situation disciplinaire et institutionnelle actuelle de la littérature française et des littératures francophones, d'autre part, peuvent paraitre ténus voir invisibles, les articles réunis dans ce numéro étayent leur évidence et leur urgence. Cinq propositions s'en dégagent qui contribuent à dédramatiser les termes du débat.

La première consiste à opérer une distinction entre les raisons légitimes d'inquiétude, fondées sur la réalité des tendances institutionnelles et des politiques éducatives, d'une part, et l'anxiété corporatiste et idéologique émanant des embrigadements disciplinaires et des présupposés culturels. La deuxième, corollaire de la première, met en garde contre le durcissement des positions et des contradictions perçues mettant en opposition un système de valeurs françaises classiques synonyme, pour certains, de qualité et de pérennité, pour d'autres, de conformisme conservateur, d'un côté, et la vague contemporaine et francophone, marquant un effet de mode fâcheux, mais éphémère, aux yeux des uns, ou une révolution nécessaire, légitime et salutaire. À la menace réelle d'une dévalorisation du français, d'abord, en tant que langue de communication et de culture et, ensuite, en tant que lieu d'articulation multiple du savoir pratique avec les disciplines scientifiques, il est vital d'apporter des solutions concrètes qui informent les approches pédagogiques tout en renouvelant les cadres de la pensée théorique - c'est notre troisième proposition. Les perspectives multidisciplinaires et transdisciplinaires ainsi que le dialogue actif et systématique entre les textes de différentes époques et de régions diverses favorisent la pluralisation des modèles critiques et herméneutiques. Cependant, un quatrième point à retenir porte sur la nécessité de reconnaittre et de cultiver les spécificités locales, pour dépasser la conception d'un français globalement uniforme et d'une production littéraire francophone qui se contenterait d'élargir à l'échelle mondiale les formes et les valeurs prescrites par le centre. Ce qui nous amène au dernier point: l'appel à adopter une attitude hospitalière et cosmopolitique à l'égard de notre objet qui permette de sortir des retranchements identitaires et de la politique du ressentiment afin de construire un espace de solidarité autant idéale que matérielle autour d'une communauté linguistique et littéraire. 


\section{Bibliographie}

Appadurai, Arjun. Après le colonialisme. Les conséquences culturelles de la globalisation, Françoise Bouillot tr., Paris : Payot, 2001.

Appiah, Anthony. The Ethics of Identity. Princeton, NJ: Princeton University Press, 2005.

Ashcroft, Bill; Griffith, Gareth; Helen Tiffin. The Postcolonial Study Reader. London: Routledge, 2006.

Balibar, Étienne. "Cosmopolitisme et internationalisme: deux modèles, deux héritages. » Philosophie politique et horizon cosmopolitique (2004): 37-65.

Bénichou, Paul. Le Sacre de l'écrivain 1750-1830. Essai sur l'avènement d'un pouvoir spirituel laïque dans la France moderne, Paris: José Corti, 1973.

Cheah, Pheng. Inbuman Conditions: On Cosmopolitanism and Human Rights. Cambridge, MA: Harvard University Press, 2006.

"What Is a World? On World Literature as World-Making Activity." Daedalus 137.3 (2008): 26-38.

Edmundson, Mark. Why Teach : In Defense of a Real Education. New York: Bloomsbury. 2013.

Gans, Eric. «Qu'est-ce que la littérature, aujourd'hui? » New Literary History, 38-1 (2007): 33-41.

McDonald, Christie; Rubin Suleiman Susan (eds). French Global: A New Approach to Literary History. New York: Columbia University Press, 2010.

Méchoulan, Éric. Le Livre avalé. De la littérature entre mémoire et culture, Montréal: Presses Universitaires de Montréal, 2004.

Moudileno, Lydie. «Littérature et postcolonie. » Africultures, May 1 (2000), http://www.africultures.com/php/index.php?nav $=$ article\&no $=13$ 59. Web.

Panaité, Oana. Des littératures-mondes en français. Écritures singulières, poétiques transfrontalières dans la prose contemporaine. Amsterdam, New York: Rodopi, 2012.

«La Querelle des bibliothèques ou la gêne de la critique française face à la littérature en français. » Nouvelles Écritures francophones, 28-1 (2013): 145-161.

Rosello, Mireille. «Unhoming Francophone Studies: A House in the Middle of the Current.» Yale French Studies «French and Francophone. The Challenge of Expanding Horizons », 103 (2003): 123-132.

Simon, Zach. «In Defense of French.» The Huffington Post. 4 April. 2014. 23 July. 2014. Web. 
Sloterdijk, Peter. «Règles pour le parc humain. » Christiane Haack trad. Le Monde des Débats, octobre 1999, i-viii.

Touret, Michèle (ed). Histoire de la littérature française du XX siècle, tome II après 1940, Rennes: Presses Universitaires de Rennes, 2008. 